\title{
研究简报
}

\section{非线性不适定问题的最大熵方法 *}

金其年 侯宗义

(复旦大学数学研究所, 上海 200433)

\section{关镜词非线性不适定问题 最大嫡方法 稳定性 收敛性}

很多数学物理问题可化为求非线性算子方程

$$
F(f)=g
$$

的满足 $f \geqslant 0$ 的解, 其中 $F: D(F) \subset \mathrm{L}^{1}(\Omega) \rightarrow \mathrm{L}^{2}(\Omega)$ 为非线性算子, 定义域 $D(F) \subset \mathrm{L}_{+}^{1}(\Omega):=$ $\left\{f \in L^{1}(\Omega) \mid f \geqslant 0\right.$ a. e. 在 $\Omega$ 上\}, 并且 $\Omega$ 为 $\mathbb{R}^{n}$ 中可测集. 例如, 在问题

$$
\begin{gathered}
-u_{x x}+a u=h, \quad x \in(0,1), \\
u(0)=g_{1}, \quad u(1)=g_{2}
\end{gathered}
$$

中, 考虑由 $u$ 的观察值 $u(x), x \in(0,1)$ 来确认参数 $a$, 其中 $h \in \mathrm{L}^{2}([0,1])$ 并且 $g_{1}, g_{2}$ 为实 数. 众所周知, 当 $a \in\left\{a \in \mathrm{L}^{1}([0,1]) \mid a \geqslant 0\right.$ a.e. 在 $[0,1]$ 上 $\}$, 问题 (2), (3) 有唯一解 $u=$ $u(a) \in \mathrm{H}^{1}([0,1]) \subset \mathrm{L}^{1}([0,1])$. 定义非线性算子 $F$ 为

$$
F: D(F) \subset \mathrm{L}^{1}([0,1]) \mapsto \mathrm{L}^{2}([0,1]), a \mapsto u(a),
$$

其中 $D(F)=\left\{a \in \mathrm{L}^{1}([0,1]) \mid a \geqslant 0\right.$ a.e. 在 $[0,1]$ 中 $\}$, 于是确认 $a$ 的问题便化为解问题 (1).

我们感兴趣于问题 (1) 为不适定的情形, 即 (1)式的解缺乏对数据的连续依赖性. 对不适 定问题必须进行正则化处理. 传统的方法是 Tikhonov 正则化 ${ }^{[1]}$. 近年来, 人们将嫡引入到不 适定问题的研究中, 发展了最大嫡方法 ${ }^{[2 \sim 6]}$, 并发现在某些问题中用最大嫡方法有独特的效 果 ${ }^{[2]}$. 现有的研究仅限于线性问题, 本文将最大嫡方法拓展到非线性不适定问题的研究中.

记

$$
\begin{aligned}
& E\left(f^{*}, \Omega\right)=\left\{f \in \mathrm{L}_{+}^{1}(\Omega) \mid \int_{\Omega} f \log \frac{f}{f^{*}} \mathrm{~d} x<\infty\right\}, \\
& E_{M}\left(f^{*}, \Omega\right)=\left\{f \in \mathrm{L}_{+}^{1}(\Omega) \mid \int_{\Omega} f \log \frac{f}{f^{*}} \mathrm{~d} x \leqslant M\right\},
\end{aligned}
$$

其中 $f^{*} \in \mathrm{L}_{+}^{1}(\Omega)$ 为一取定函数并满足 $0<k \leqslant f^{*}(x) \leqslant K$ a.e. 在 $\Omega$ 上, $k, K, M$ 为正常数.

首先引入 $f^{*}$ - 最大嫡解的概念.

定义 1 称 $f_{0} \in \mathrm{L}_{+}^{1}(\Omega)$ 为问题 (1) 的 $f^{*}$ - 最大嫡解, 如果

$$
F\left(f_{0}\right)=g \text { 并且 } \int_{\Omega} f_{0} \log \frac{f_{0}}{f^{*}} \mathrm{~d} x=\inf \left\{\int_{\Omega} f \log \frac{f}{f^{*}} \mathrm{~d} x \mid F(f)=g\right\} \text {. }
$$

1995-10-05 收稿, 1996-01-03 收修改稿

* 国家自然科学基金资助项目 
以下总假定 (1) 的 $f^{*}$ - 最大嫡解存在. 研究问题 (1) 的最大嫡方法就是用最小化问题

$$
\min _{f \in D(F)}\left\{\left\|F(f)-g_{\delta}\right\| L_{L^{2}(\Omega)}^{2}+\alpha \int_{\Omega} f \log \frac{f}{f^{*}} \mathrm{~d} x\right\}
$$

的解 $f_{\alpha}^{\delta}$ 来逼近 (1) 式的解, 其中 $g_{\delta}$ 为 $g$ 的测量数据, $\left\|g_{\delta}-g\right\|_{L^{2}(\Omega)} \leqslant \delta, \alpha>0$ 称为正则化参 数, $f_{\alpha}^{\delta}$ 称为最大摘正则化解. 本文将研究最大嫡方法的稳定性和最大嫡正则化解 $f_{\alpha}^{\delta}$ 的收玫 性问题. 我们总假定 $F$ 是弱闭的.

引理 1 对每个正数 $M$, 集合 $E_{M}\left(f^{*}, \Omega\right)$ 为 $\mathrm{L}^{1}(\Omega)$ 中的弱紧集.

证 由 Vallée-Poussin 准则 ${ }^{[7]}$ 立得 $E_{M}\left(f^{*}, \Omega\right)$ 的弱列紧性. 剩下只需证 $E_{M}\left(f^{*}, \Omega\right)$ 在 $\mathrm{L}^{1}(\Omega)$ 中为弱闭的. 注意到 $E_{M}\left(f^{*}, \Omega\right)$ 为 $\mathrm{L}^{1}(\Omega)$ 中凸集, 于是只需证 $E_{M}\left(f^{*}, \Omega\right)$ 为 $\mathrm{L}^{1}(\Omega)$ 中的闭集. 设 $\left\{f_{n}\right\} \subset E_{M}\left(f^{*}, \Omega\right), f \in \mathrm{L}^{1}(\Omega)$ 使得 $\left\|f_{n}-f\right\|_{\mathrm{L}^{1}(\Omega)} \rightarrow 0(n \rightarrow \infty)$, 于是在 $\Omega$ 上 a.e. 成立 $f_{n} \rightarrow f$, 因此在 $\Omega$ 上. a.e. 成立 $f_{n} \log \frac{f_{n}}{f^{*}}+\frac{f^{*}}{\mathrm{e}} \rightarrow f \log \frac{f}{f^{*}}+\frac{f^{*}}{\mathrm{e}}$. 但 $f_{n} \log \frac{f_{n}}{f^{*}}+\frac{f^{*}}{\mathrm{e}}$ 在 $\Omega$ 上非负, 于是由 Fatou 引理知 $\int_{\Omega}\left(f \log \frac{f}{f^{*}}+\frac{f^{*}}{\mathrm{e}}\right) \mathrm{d} x \leqslant \liminf _{n \rightarrow \infty} \int_{\Omega}\left(f_{n} \log \frac{f_{n}}{f^{*}}+\frac{f^{*}}{\mathrm{e}}\right) \mathrm{d} x$, 即 $\int_{\Omega}$ $f \log \frac{f}{f^{*}} \mathrm{~d} x \leqslant \liminf _{n \rightarrow \infty} \int_{\Omega} f_{n} \log \frac{f_{n}}{f^{*}} \mathrm{~d} x$, 这说明 $f \in E_{M}\left(f^{*}, \Omega\right)$.

引理 2 泛函 $f \mapsto \int_{\Omega} f \log \frac{f}{f^{*}} \mathrm{~d} x$ 是弱下半连续的.

证 设 $\left\{f_{n}\right\} \subset E\left(f^{*}, \Omega\right)$ 并且在 $\mathrm{L}^{1}(\Omega)$ 中 $f_{n} \stackrel{\text { 弱 }}{\longrightarrow} f(n \rightarrow \infty)$. 记 $M=\liminf _{n \rightarrow \infty} \int_{\Omega} f_{n} \log \frac{f_{n}}{f^{*}} \mathrm{~d} x$, 则 $\forall \varepsilon>0$, 存在子序列 $\left\{f_{n_{k}}\right\}$ 使得 $f_{n_{k}} \in E_{M+\varepsilon}\left(f^{*}, \Omega\right)$ 并且在 $L^{1}(\Omega)$ 中 $f_{n_{k}} \stackrel{\text { 弱 }}{\longrightarrow} f$, 于是由引理 1 知 $f \in E_{M+c}\left(f^{*}, \Omega\right)$. 由 $\varepsilon$ 的任意性知 $f \in E_{M}\left(f^{*}, \Omega\right)$. 证毕.

下面的结果给出了最大熵正则化解的存在性.

定理 1 对每个 $\alpha>0$, 最小化问题 (4) 的解存在.

证 记 $d_{\alpha}:=\inf _{f \in D(F)}\left\{\left\|F(f)-g_{\delta}\right\|_{L^{2}(\Omega)}^{2^{2}}+\alpha \int_{\Omega} f \log \frac{f}{f^{*}} \mathrm{~d} x\right\}$, 并取序列 $\left\{f_{n}\right\} \subset D(F)$ 使 得 $\lim _{n \rightarrow \infty}\left\{\left\|F\left(f_{n}\right)-g_{\delta}\right\|_{\mathrm{L}^{2}(\Omega)}^{2}+\alpha \int_{\Omega} f_{n} \log \frac{f_{n}}{f^{*}} \mathrm{~d} x\right\}=d_{\alpha}$. 由此可知存在正常数 $M(\alpha)$ 使得 $\left\|F\left(f_{n}\right)-g_{\delta}\right\|_{\mathrm{L}^{2}(\Omega)} \leqslant M(\alpha), \int_{\Omega} f_{n} \log \frac{f_{n}}{f^{*}} \mathrm{~d} x \leqslant M(\alpha)$. 由引理 1 及 Hilbert 空间中有界集的 弱列紧性知存在 $\left\{f_{n}\right\}$ 的子序列 $\left\{f_{n_{k}}\right\}$ 及元 $f_{a}^{\delta} \in E_{M(\alpha)}\left(f^{*}, \Omega\right), \bar{g}_{\delta} \in \mathrm{L}^{2}(\Omega)$ 使得

$$
f_{n_{k}} \stackrel{\text { 弱 }}{\longrightarrow} f_{\alpha}^{\delta}\left(\text { 在 } \mathrm{L}^{1}(\Omega) \text { 中), } F\left(f_{n_{k}}\right) \stackrel{\text { 弱 }}{\longrightarrow} \bar{g}_{\delta} \text { (在 } \mathrm{L}^{2}(\Omega)\right. \text { 中). }
$$

由 $F$ 的弱闭性知 $f_{\alpha}^{\delta} \in D(F)$ 且 $F\left(f_{\alpha}^{\delta}\right)=\bar{g}_{o}$. 根据引理 2 及Hilbert空间范数的弱下半连续性知

$$
\begin{aligned}
& \left\|F\left(f_{\alpha}^{\delta}\right)-g_{\delta}\right\|_{L^{2}(\Omega)}^{2}+\alpha \int_{\Omega} f_{\alpha}^{\delta} \log \frac{f_{\alpha}^{\delta}}{f^{*}} \mathrm{~d} x \leqslant \\
& \underset{n \rightarrow \infty}{\liminf }\left\{\left\|F\left(f_{n_{k}}\right)-g_{\delta}\right\|_{L^{2}(\Omega)}^{2}+\alpha \int_{\Omega} f_{n_{k}} \log \frac{f_{n_{k}}}{f^{*}} \mathrm{~d} x\right\}=d_{\alpha},
\end{aligned}
$$

由 $d_{\alpha}$ 的定义知 $f_{\alpha}^{\delta}$ 就是最小化问题 (4)的解.

定义 $2 E\left(f^{*}, \Omega\right)$ 中一序列 $\left\{f_{n}\right\}$ 称为按嫡收玫于 $f \in \mathrm{L}_{+}^{1}(\Omega)$, 如果 


$$
\lim _{n \rightarrow \infty} \int_{\Omega} f_{n} \log \frac{f_{n}}{f^{*}} \mathrm{~d} x=\int_{\Omega} f \log \frac{f}{f^{*}} \mathrm{~d} x .
$$

引理 3 设 $\left\{f_{n}\right\} \subset E\left(f^{*}, \Omega\right), f \in E\left(f^{*}, \Omega\right)$, 在 $\mathrm{L}^{1}(\Omega)$ 中 $f_{n} \stackrel{\text { 弱 }}{\longrightarrow} f$, 并且 $f_{n}$ 按嫡收玫于 $f$, 则必有 $\lim _{n \rightarrow \infty}\left\|f_{n}-f\right\|_{L^{1}(\Omega)}=0$.

证 见文献[3]中定理 4 或文献[4]中定理 2.7.

现考虑最大嫡方法的稳定性. 当 $F$ 为线性时, 相应的结果已在文献 $[5,6]$ 中给出, 文献 [5]是在弱收玫意义下获得的, 而文献 [6]是在强收玫意义下得到的,但需要相当强的条件.

定理 2 设 $\left\{g_{n}\right\} \subset \mathrm{L}^{2}(\Omega)$ 满足 $\lim _{n \rightarrow \infty}\left\|g_{n}-g_{\delta}\right\|_{\mathrm{L}^{2}(\Omega)}=0$, 设 $\alpha>0$ 给定,记 $\left\{f_{n}\right\}$ 为最小化问题

$$
\min _{f \in D(F)}\left\{\left\|F(f)-g_{n}\right\|_{L^{2}(\Omega)}^{2}+\alpha \int_{\Omega} f \log \frac{f}{f^{*}} \mathrm{~d} x\right\}
$$

的解构成的序列, 则一定存在在 $L^{1}(\Omega)$ 中收玫的子序列 $\left\{f_{n_{k}}\right\}$, 并且 $\left\{f_{n}\right\}$ 的每个收玫子序列的 极限均为最小化问题 (4) 的解.

证 由于 $f_{n}$ 为 (5) 式的解, 有

$$
\left\|F\left(f_{n}\right)-g_{n}\right\|_{\mathrm{L}(\Omega)}^{2}+\alpha \int_{\Omega} f_{n} \log \frac{f_{n}}{f^{*}} \mathrm{~d} x \leqslant\left\|F\left(f_{\alpha}^{\delta}\right)-g_{n}\right\|_{\mathrm{L}(\Omega)}^{2}+\alpha \int_{\Omega} f_{\alpha}^{\delta} \log \frac{f_{\alpha}^{\delta}}{f^{*}} \mathrm{~d} x,
$$

从而存在正常数 $M:=M(\alpha, \delta)$ 使得

$$
\left\|F\left(f_{n}\right)-g_{n}\right\|_{\mathrm{L}^{2}(\Omega)} \leqslant M, \int_{\Omega} f_{n} \log \frac{f_{n}}{f^{*}} \mathrm{~d} x \leqslant M .
$$

于是存在 $\left\{f_{n}\right\}$ 的子序列 $\left\{f_{n_{k}}\right\}$ 及元 $f \in E_{M}\left(f^{*}, \Omega\right), g \in \mathrm{L}^{2}(\Omega)$ 使得

$$
f_{n_{k}} \stackrel{\text { 弱 }}{\longrightarrow} f\left(\text { 在 } \mathrm{L}^{1}(\Omega) \text { 中), } F\left(f_{n_{k}}\right) \stackrel{\text { 弱 }}{\longrightarrow} g\left(\text { 在 } \mathrm{L}^{2}(\Omega) \text { 中 }\right)\right. \text {, }
$$

由 $F$ 的弱闭性知 $f \in D(F), F(f)=g$. 现任取 $\bar{f} \in D(F)$, 则由引理 2 及 Hilbert 空间范数的 弱下半连续性知

$$
\begin{gathered}
\left\|F(f)-g_{\delta}\right\|_{\mathrm{L}^{2}(\Omega)}^{2}+\alpha \int_{\Omega} f \log \frac{f}{f^{*}} \mathrm{~d} x \leqslant \liminf _{k \rightarrow \infty}\left\{\left\|F\left(f_{n_{k}}\right)-g_{n_{k}}\right\|_{\mathrm{L}^{2}(\Omega)}^{2}+\alpha \int_{\Omega} f_{n_{k}} \log \frac{f_{n_{k}}}{f^{*}} \mathrm{~d} x\right\} \leqslant \\
\lim _{k \rightarrow \infty}\left\{\left\|F(\bar{f})-g_{n_{k}}\right\|_{\mathrm{L}(\Omega)}^{2{ }^{2}(\Omega)}+\alpha \int_{\Omega} \bar{f} \log \frac{\bar{f}}{f^{*}} \mathrm{~d} x\right\}= \\
\left\|F(\bar{f})-g_{\delta}\right\|_{\mathrm{L}(\Omega)}^{2{ }^{2}(\Omega)}+\alpha \int_{\Omega} \bar{f} \log \frac{\bar{f}}{f^{*}} \mathrm{~d} x .
\end{gathered}
$$

此即说明 $f$ 为最小化问题 (4) 的解.

剩下需证 $\lim _{k \rightarrow \infty}\left\|f_{n_{k}}-f\right\|_{L^{1}(\Omega)}=0$. 由引理 3 知只需证 $\int_{\Omega} f \log \frac{f}{f^{*}} \mathrm{~d} x=\lim _{k \rightarrow \infty} \int_{\Omega} f_{n_{k}} \log \frac{f_{n_{k}}}{f^{*}} \mathrm{~d} x$.

而由引理 2 知 $\int_{\Omega} f \log \frac{f}{f^{*}} \mathrm{~d} x \leqslant \liminf _{k \rightarrow \infty} \int_{\Omega} f_{n_{k}} \log \frac{f_{n_{k}}}{f^{*}} \mathrm{~d} x$, 因此只需证

$$
C_{0}:=\int_{\Omega} f \log \frac{f}{f^{*}} \mathrm{~d} x \geqslant \limsup _{k \rightarrow \infty} \int_{\Omega} f_{n_{k}} \log \frac{f_{n_{k}}}{f^{*}} \mathrm{~d} x:=C .
$$

假设 $C_{0}<C$. 易知存在 $\left\{f_{n_{k}}\right\}$ 的子序列, 为简单起见仍记为 $\left\{f_{n_{k}}\right\}$, 使得

$$
\left\|F\left(f_{n_{k}}\right)-g_{n_{k}}\right\|{ }_{\mathrm{L}}^{2}(\Omega) \geqslant \liminf _{k \rightarrow \infty}\left\|F\left(f_{n_{k}}\right)-g_{n_{k}}\right\|_{L^{2}(\Omega)}^{2}-\frac{\alpha\left(C-C_{0}\right)}{4},
$$




$$
\int_{\Omega} f_{n_{k}} \log \frac{f_{n_{k}}}{f^{*}} \mathrm{~d} x \geqslant \limsup _{k \rightarrow \infty} \int_{\Omega} f_{n_{k}} \log \frac{f_{n_{k}}}{f^{*}} \mathrm{~d} x-\frac{C-C_{0}}{4} .
$$

于是有

$$
\begin{aligned}
& \left\|F(f)-g_{\delta}\right\|_{L^{2}(\Omega)}^{22}+\alpha \int_{\Omega} f \log \frac{f}{f^{*}} \mathrm{~d} x \leqslant \\
& \liminf _{k \rightarrow \infty}\left\|F\left(f_{n_{k}}\right)-g_{n_{k}}\right\|_{L^{2}(\Omega)}^{2}+\alpha \limsup _{k \rightarrow \infty} \int_{\Omega} f_{n_{k}} \log \frac{f_{n_{k}}}{f^{*}} \mathrm{~d} x-\alpha\left(C-C_{0}\right) \leqslant \\
& \left\|F\left(f_{n_{k}}\right)-g_{n_{k}}\right\|_{L^{2}(\Omega)}^{2}+\alpha \int_{\Omega} f_{n_{k}} \log \frac{f_{n_{k}}}{f^{*}} \mathrm{~d} x-\frac{\alpha\left(C-C_{0}\right)}{2} .
\end{aligned}
$$

注意到 $\lim _{k \rightarrow \infty}\left\|g_{n_{k}}-g_{\delta}\right\|_{L^{2}(\Omega)}=0$, 故当 $k$ 充分大时有

$$
\left\|F(f)-g_{n_{k}}\right\|_{L^{2}(\Omega)}^{2}+\alpha \int_{\Omega} f \log \frac{f}{f^{*}} \mathrm{~d} x<\left\|F\left(f_{n_{k}}\right)-g_{n_{k}}\right\|_{L^{2}(\Omega)}^{2}+\alpha \int_{\Omega} f_{n_{k}} \log \frac{f_{n_{k}}}{f^{*}} \mathrm{~d} x,
$$

这与 $f_{n_{k}}$ 的定义矛盾.

最后给出最大嫡正则化解 $f_{\alpha}^{\delta}$ 的收玫性.

定理 3 设 $g_{\delta}$ 满足 $\left\|g_{\delta}-g\right\|_{L^{2}(\Omega)} \leqslant \delta$, 并设 $f_{\alpha}^{\delta}$ 为最大嫡正则化解, 设 $\alpha:=\alpha(\delta)$ 的选取 满足 $\alpha(\delta) \rightarrow 0, \frac{\delta^{2}}{\alpha(\delta)} \rightarrow 0$ (当 $\left.\delta \rightarrow 0\right)$, 则 $\left\{f_{\alpha(\delta)}^{\delta}\right\}$ 具有在 $\mathrm{L}^{1}(\Omega)$ 中收玫的子序列, 并且每个收玫子 序列的极限均为 (1) 的 $f^{*}$ - 最大嫡解. 进一步地, 如果 (1) 式的 $f^{*}$-最大嫡解 $f_{0}$ 唯一, 则

$$
\lim _{\delta \rightarrow 0}\left\|f_{\alpha(\delta)}^{\delta}-f_{0}\right\|_{L^{1}(\Omega)}=0 \text {. }
$$

证 由 $f_{\alpha(\delta)}^{\delta}$ 的定义有

$$
\left\|F\left(f_{\alpha(\delta)}^{\delta}\right)-g_{\delta}\right\|_{\mathrm{L}^{2}(\Omega)}^{2}+\alpha(\delta) \int_{\Omega} f_{\alpha(\delta)}^{\delta} \log \frac{f_{\alpha(\delta)}^{\delta}}{f^{*}} \mathrm{~d} x \leqslant \delta^{2}+\alpha(\delta) \int_{\Omega} f_{0} \log \frac{f_{0}}{f^{*}} \mathrm{~d} x,
$$

由此并注意到 $\alpha(\delta)$ 的选取知存在正常数 $M>0$ 使得

$$
\int_{\Omega} f_{\alpha(\delta)}^{\delta} \log \frac{f_{\alpha(\delta)}^{\delta}}{f^{*}} \mathrm{~d} x \leqslant M \text { 并且 } \quad \lim _{\delta \rightarrow 0}\left\|F\left(f_{\alpha(\delta)}^{\delta}\right)-g_{\delta}\right\|_{L^{2}(\Omega)}=0 .
$$

余下的可类似于定理 2 的证明来完成.

\section{参考 文 献}

1 Tikhonov A N, Arsenin V Y. Solutions of Ill-Posed Problems. Washington D C: Winstion, 1977

2 Skilling K ed. Maximum Entropy and Bayesian Methods. Dordrecht: Kluwer, 1989

3 Amato U, Hughes W. Maximum entropy regularization of Fredholm integral equation of the first kind. Inverse Problems, $1991,7(6): 793 \sim 808$

4 Borwein J M, Lewis C S. Convergence of best entropy estimates. STAM J Optimization, 1991, 1(1):191 205

5 Klaus M, Smith R T. A Hilbert space approach to maximum entropy reconstruction. Math Meth Appl Sci, 1988, 10(4):397 $\sim 406$

6 Engl H W, Landl G. Convergence rates for maximum entropy regularization. SIAM J Numer Anal, 1993, 30(5):1 509 1536

7 Krasnoselskii M A. Integral Operators in Spaces of Summable Functions. The Netherlands: Noordhoff International Publishing, 1976 